\title{
IMPACTO DE LA INTERCONEXIÓN DE MERCADOS ELÉCTRICOS TRANSFRONTERIZOS EN LA EXPANSIÓN DE LOS PARQUES DE GENERACIÓN DE PERÚ Y ECUADOR
}

\author{
IMPACT OF THE INTERCONNECTION OF CROSS-BORDER \\ ELECTRICITY MARKETS IN THE EXPANSION OF THE GENERATION \\ PARKS OF PERU AND ECUADOR
}

\author{
Fabiola Jara- Caytuiro ${ }^{{ }^{*} \mathbb{D}}$, Jaime E. Luyo- Kuong ${ }^{1(i)}$ \\ 'Universidad Nacional de Ingeniería, Facultad de Ingeniería Mecánica, Lima, Perú
}

Recibido (Received): 05 / 03/2020 Aceptado (Accepted): 18/ 02 / 2021

\begin{abstract}
RESUMEN
En los últimos años a nivel internacional se están llevando a cabo la interconexión de mercados eléctricos, la cual posibilita la optimización del uso de recursos energéticos y mejora la confiabilidad de los sistemas. Este proceso de integración eléctrica genera una redistribución de los beneficios entre los agentes, lo cual también genera nuevos problemas para la coordinación y regulación, por lo cual existe la necesidad de diseñar nuevas políticas de regulación basadas en análisis de los impactos de la interconexión de mercados transfronterizos. El objetivo del presente artículo es mejorar la comprensión de los efectos que tendrá la interconexión en parámetros como: la evolución del parque de generación electricidad en cada sistema eléctrico, la incorporación de centrales hidroeléctricas de gran tamaño especialmente en países que cuenten con complementariedad hídrica y la incorporación de centrales eléctricas a base de recursos energéticos renovables no convencionales. Para ello, se ha estructurado un modelo que hace uso de la teoría de dinámica de sistemas y se analizó el caso de la interconexión Perú - Ecuador, encontrando que las políticas de estado previamente aplicadas en ambos países han originado que los costos marginales de sus sistemas sean relativamente bajos, con lo cual no ingresaría nueva generación en el corto plazo; asimismo, se concluyó que aunque expandir la capacidad hidroeléctrica puede parecer la mejor solución dada la complementariedad hídrica entre ambos países, los bajos costos marginales hacen que las primeras centrales a ser instaladas sean a base de gas natural.
\end{abstract}

Palabras Clave: Interconexión, mercado de electricidad, políticas de regulación, simulación, complementariedad

\section{ABSTRACT}

In the last years at international level the interconnection of electrical markets is being carried out, which makes it possible to optimize the use of energy resources and improve the reliability of the systems. This process of electrical integration generates a redistribution of benefits among agents, which also generates new problems for coordination and regulation, so there is a need to design new regulation policies based on analysis of the impacts of market interconnection. cross-border The objective of this article is to improve the understanding of the effects that interconnection will have on parameters such as: the evolution of the electricity generation park in each electrical system, the incorporation of large hydroelectric power plants especially in countries that have water complementarity and incorporation of power plants based on non-conventional renewable energy resources. To do this, a model has been structured that makes use of the theory of systems dynamics and the case of the PeruEcuador interconnection was analyzed, finding that the state policies previously applied in both countries have caused that the marginal costs of their systems they are relatively low, so that new generation would not enter in the short term; It was also concluded that although expanding hydroelectric capacity may seem the best solution given the water complementarity between the two countries, low marginal costs mean that the first plants to be installed are based on natural gas.

Keywords: Interconnection, electricity market, regulation policies, simulation, complementarity

\section{INTRODUCCION}

Es América Latina como en diferentes partes del mundo, muchos países han decidido integrar sus sistemas de electricidad con

* Corresponding author.:

E-mail: fabiolajaraca@gmail.com objetivos de: Ganar eficiencia en el uso de sus recursos, mejorar la seguridad de suministro, reducirlos costos, entre otros. El logro de estos objetivos depende de una expansión adecuada de la capacidad de generación y transmisión, ya que se busca aprovechar de forma óptima los recursos energéticos aliviando así los picos de demanda. El comercio de electricidad se da 
a través de un esquema de acoplamiento de mercado, para que dos regiones obtengan beneficios de costo y capacidad de manera óptima, lo cual está sujeto a límites en la capacidad de interconexión, dichos esquemas se han aplicado en sistemas liberalizados a través de modificaciones en el diseño del mercado y en el marco regulatorio [1].

Sin embargo, no hay acuerdo sobre cuáles son las políticas correctas para mejorar la fiabilidad, ya que poca capacidad de generación pondría en peligro la seguridad del suministro y podría aumentar el poder del mercado de las empresas de generación; pero, por otro lado, demasiada capacidad costaría más de lo que la gente está dispuesta a pagar y esto afecta la capacidad de recuperar los costos de inversión y se desincentiva nuevas inversiones en generación y transmisión. Asimismo, el proceso de liberalización de los mercados de electricidad, crea desafíos para la planificación de la expansión, ya que se requiere un alto nivel de coordinación entre los agentes para desarrollar un sistema en el que las capacidades de generación y transmisión sean conjuntamente óptimas. Además, las inversiones en generación y transmisión no solo son interdependientes, sino que también se ven afectadas por el marco regulatorio. Por lo tanto, se deben enviar señales económicas para coordinar ambas decisiones de inversión [2].

En la actualidad muchos países con planes de interconexión intentan resolver los problemas de la remuneración de la capacidad de manera unilateral, y estos difieren de un país a otro considerablemente, ya que toman en cuenta factores de acuerdo a su realidad nacional, no teniendo en cuenta un análisis conjunto de los mercados que se planea interconectar, lo cual afectaría a la interconexión ya que los mecanismos de capacidad no coordinados pueden distorsionar el comercio transfronterizo [3]. En este caso, los flujos y precios de la electricidad pueden verse afectados negativamente por estas diferencias, por esta razón se debe implementar políticas coordinadas, para que no se presente el caso de que un mercado subsidie al otro. Por lo tanto, los mecanismos de capacidad de mercado deben abrirse a la participación transfronteriza explícita para minimizar las distorsiones en el comercio transfronterizo, así garantizar la reducción de los costos de suministro a largo plazo [4].

El objetivo de la interconexión transfronteriza de electricidad es principalmente la optimización en el uso de recursos lo cual conlleva una reducción de costos de generación, sin embargo, los mecanismos de capacidad generan inquietudes acerca de la confiabilidad, debido a que las rentas de congestión de la interconexión se dividen entre ambos países, y esto puede originar que las inversiones sean difíciles asumir para un generador exportador en el país exportador ya que estaría asumiendo una obligación que esta fuera de su control en el país importador[5].

Estos eventos afectan la eficiencia de la interconexión a corto y a largo plazo por eso es importante estudiarlos mediante diferentes escenarios, teniendo en cuenta: i) los mecanismos de capacidad, ii) la participación en el mercado interconectado y iii) características propias del mercado nacional.

El objetivo de la presente investigación es mejorar la comprensión de los efectos que tendrá la interconexión de mercados eléctricos transfronterizos en los siguientes puntos:

- La evolución del parque de generación electricidad en cada sistema eléctrico.

- La incorporación de centrales hidroeléctricas de gran tamaño; en países que cuenten con complementariedad hídrica.

- La incorporación de centrales eléctricas a base de recursos energéticos renovables no convencionales.

Para alcanzar dicho objetivo se ha desarrollado el modelo propuesto por Jara et al [6], el cual hace uso de la teoría de dinámica de sistemas para interrelacionar:

i. Un modelo de expansión de la generación con un horizonte de simulación de largo plazo el cual sea suficiente para observar la evolución de las diferentes tecnologías de generación y cuenta con una resolución mensual que permita observar las variaciones estacionales de la oferta debidas a la hidrología.

ii. Un modelo de despacho económico de corto plazo para determinar el impacto en los costos marginales que afectarán la rentabilidad de los nuevos proyectos de generación, así como de las líneas de interconexión.

El análisis realizado se centra en el caso particular de Perú y Ecuador. La Sección 2 presenta los antecedentes de las iniciativas parala interconexión de ambos países y las principales características de sus sistemas eléctricos que puedan tener un impacto en nuestros resultados, asimismo, presenta una breve revisión de la literatura, en la sección 3 se hace una descripción del modelo empleado, en la sección 4 se presentan los resultados del análisis realizado para el caso de la interconexión entre Perú y Ecuador; Finalmente, en sección 5 se presentan las conclusiones.

\section{ANTECEDENTES}

La interconexión transfronteriza de mercados eléctricos se ha estudiado desde hace algunas décadas con enfoques que van desde análisis técnicos, económicos y políticos. Para realizar estos análisis se 
simularon mercados eléctricos específicos, ya que cada país tiene políticas específicas que responden a su realidad.

Una de las dificultades encontradas en el análisis de los efectos transfronterizos es la gran cantidad de posibles influencias, como la cantidad y el tamaño del mercado de los países considerados. Además, los niveles de competencia y los diseños de mercado respectivos pueden influir en los resultados [7]. Por lo tanto, es difícil sacar conclusiones generales. Este hecho podría servir como una explicación de por qué la literatura se centra predominantemente en un escenario de mercado único, y la investigación sobre los efectos indirectos de los mecanismos de remuneración de la capacidad está rezagada [8]. Sin embargo, sin un marco teórico sólido sobre los efectos transfronterizos, garantizar la adecuación de la generación a nivel regional de manera eficiente sigue siendo un desafío importante [9], Esto se complica aún más por el hecho de que los efectos transfronterizos pueden surgir de manera no lineal [10].

Una pregunta que se examina con frecuencia en la literatura es si la figura de un "free rider" ocurre si un país vecino introduce un Mecanismos de Remuneración de capacidad (MRC), Por ejemplo, Bhagwat et al. [11]. estudian los efectos transfronterizos en dos áreas simétricas del mercado que difieren solo en su diseño. Mientras que un mercado de solo energía (ME) no limita la eficacia del mercado de capacidad o la reserva estratégica (RE) vecina, o viceversa, se pueden observar dos efectos: por un lado, los consumidores en el ME son independientes sobre los consumidores en el mercado vecino donde se implementa MRC. Por otro lado, la dependencia de la ME de los mercados vecinos está aumentando. De manera similar, Meyer y Gore en [7] encuentran que la implementación unilateral de un $M R C$, ya sea en forma de opciones de confiabilidad o un $\mathrm{RE}$, debilita los incentivos a la inversión en el mercado vecino. Cepeda y Finon en [12] analizan los efectos transfronterizos de tres diseños de mercado diferentes (ME, ME con precio tope, mercado de capacidad a futuro). En donde encuentran que, a largo plazo, el área de mercado con una ME no se beneficia del área de mercado adyacente donde se implementa un mercado de capacidad a futuro con precio tope, e incluso pueden surgir externalidades negativas en forma de un precio promedio más alto y baja confiabilidad.

Lorenczik en [8] observa que el efecto negativo de los precios tope se intensifica si un mercado está conectado a mercados vecinos $\mathrm{y}$, por lo tanto, la capacidad de generación y el bienestar disminuyen aún más. Sin embargo, de forma contraria, los precios máximos nacionales no parecen tener un efecto adverso significativo en los países vecinos. Al contrario de otros estudios, se afirma que los pagos por capacidad no ejercen un efecto positivo significativo sobre la seguridad del suministro en los países vecinos.

No solo entre un mercado con y sin MRC, pueden ocurrir efectos indirectos, sino también entre mercados con diferentes MRC. En un escenario donde se introduce un RE en un mercado y un mercado de capacidad en el otro, Bhagwat et al. En [11] observan efectos indirectos negativos del mercado de capacidad en la RE que dan como resultado, por ejemplo, un menor margen de reserva en el mercado con la RE. Elberg en [13] investiga dos áreas de mercado simétricas en las que se han implementado pagos de RE o de capacidad. De forma aislada, ambos mecanismos conducen a un resultado eficiente. Sin embargo, en una evaluación combinada, el RE muestra peores resultados debido a los efectos de redistribución, ya que el bienestar del consumidor disminuye en el área del RE, mientras que aumenta en el área adyacente.

En algunos casos, los MRC también se investigan en estudios de casos del mundo real. Por ejemplo, Ochoa y Gore en [14] investigan el bienestar y la seguridad del suministro en el mercado finlandés de electricidad considerando los posibles beneficios y riesgos derivados de la conexión con el mercado ruso. En caso de que las importaciones de electricidad de Rusia estuvieran disponibles de manera confiable, se recomendaría la expansión de las capacidades de transmisión. Sin embargo, como su confiabilidad es dudosa, se recomienda desarrollar capacidades de generación nacional y mantener un RE. En otro análisis, Ochoa y van Ackere [15] examinan los efectos transfronterizos en Colombia - Ecuador y Francia - Gran Bretaña. Concluyen que los beneficios potenciales están fuertemente vinculados a la complementariedad del mercado y que las medidas de política para explotar estos beneficios sin distorsionar las señales del mercado deben evaluarse cuidadosamente, especialmente si existen grandes capacidades de almacenamiento estacional, que podrían usarse ampliamente durante situaciones de escasez en los países vecinos. país y posteriormente no están disponibles para uso nacional. En un estudio de seguimiento, Ochoa y van Ackere en [16] analizan una vez más los mercados de Colombia - Ecuador y descubren que los tamaños relativos del mercado y el tamaño de las capacidades de transmisión tienen una influencia significativa en los beneficios transfronterizos potenciales.

Uno de los desafíos clave restantes en la evaluación de la adecuación de la generación es evaluar la contribución de los países vecinos para evitar el exceso o la falta de capacidad. Mastropietro en [4] investigan las posibilidades para eliminar las barreras que impiden que los participantes extranjeros en Europa participen en mecanismos de capacidad externa sin reducir la 
eficiencia a corto plazo del mercado eléctrico. Proponen que las capacidades deben adquirirse a través de subastas zonales, que tienen en cuenta la capacidad máxima de transmisión de la interconexión, y que no se debe permitir que las capacidades participen en diferentes MRC nacionales. Finon en [5] investiga las diferencias entre la participación transfronteriza explícita e implícita. A largo plazo, afirma que excluir a los participantes transfronterizos no da como resultado una eficiencia significativamente menor ni un efecto distorsionador en la competencia. Sin embargo, desde una perspectiva europea, la consideración explícita de las capacidades puede ser ventajosa. Además, se puede observar que la introducción de un MRC en un país vecino aumenta considerablemente la presión para introducir un mecanismo nacional, a fin de proteger el mercado contra posibles consecuencias perjudiciales [11] y [17]. Otra posibilidad es centrarse en la coordinación supranacional como se indica en [18], [19] y [20]. En este punto, debe enfatizarse que la introducción descoordinada de MRC en un sistema de eléctrico continental estrechamente interconectado, puede distorsionar las señales de precios e incluso perjudicar la seguridad del suministro en un mercado vecino. Sin embargo, a pesar de la investigación existente, los efectos transfronterizos de los MRC aún no se han explorado por completo y, en particular, el impacto en mercados del mundo real estrechamente conectados sigue siendo en gran medida desconocido.

\subsection{INTERCONEXIÓN PERÚ - ECUADOR}

El Acuerdo de Cuenca: Suscrito en noviembre de 2012, mediante el cual ambos países acordaron dar inicio al proyecto para la construcción y puesta en marcha de una nueva interconexión eléctrica entre Ecuador y Perú en un nivel de voltaje de $500 \mathrm{kV}$, que tendrá el carácter de Binacional, y en calidad de tal, será ejecutado conjuntamente respetando los procedimientos $y$ normativa interna de cada país. Las que al final del encuentro concretaron lo siguiente:

- Acuerdo Interinstitucional entre el OSINERGMIN, y la SUNAT del Perú y la ARCH de Ecuador.

- Acuerdo de Piura para la Construcción para la Línea de Interconexión Internacional Perú - Ecuador a nivel de $500 \mathrm{KV}$.

Esta interconexión forma parte de un plan de integración del sistema eléctrico que incluye los países de: Perú, Ecuador, Chile, Colombia y Bolivia. De estas interconexiones, la que funciona continuamente es la de Colombia y Ecuador, están interconectados por cuatro líneas de transmisión, con una capacidad de 500 MW, y están comercializando activamente electricidad. Esto se logró gracias a varios estudios tomando en cuenta las características particulares de cada sistema.
Los mercados de electricidad de Perú y Ecuador tienen tecnologías similares, mecanismos de despacho y tarifas de transmisión, entre otros, esto facilita la integración bajo acoplamiento de mercado. Sin embargo, estos países tienen reformas para garantizar la seguridad del suministro sin depender de las importaciones de sus vecinos, lo que puede afectar el desarrollo posterior de la interconexión, así como los beneficios que se logran en el proceso de interconexión, a inicios de siglo ambos países se dirigían hacia la liberalización de sus mercados eléctricos. Sin embargo, la situación actual es diferente. Mientras que el mercado eléctrico peruano está liberalizado en su segmento de generación, en Ecuador la responsabilidad de las inversiones en generación las tiene el estado.

Planificar la expansión sin tener en cuenta las interconexiones, es importante, ya que se implementa un mercado de capacidad, por lo que el regulador puede influir en la capacidad de generación disponible a través de este mecanismo. Teniendo en cuenta que es poco probable que el regulador considere las importaciones tan confiables como la generación dentro de país; por lo tanto, el mercado de capacidad probablemente tenderá a garantizar la seguridad del suministro con solo generación nacional $\mathrm{Si}$ bien las políticas implementadas en Perú y Ecuador están dirigidas a garantizar la independencia de capacidad de los países vecinos, se tiene un incentivo para integrar sus sistemas eléctricos: estos países tienen un importante potencial hidrológico lo cuales son tienen un grado de complementariedad, y esta ventaja puede comparativa puede ser aprovechado en la interconexión.

La Tabla I, presenta el parque de generación actualmente instalado en cada uno de los países, así como el margen de reserva, relativamente alto que ambos tienen.

TABLA I

Parque de generación instalado en cada país

\begin{tabular}{l|rr}
\hline \multicolumn{1}{c|}{ Tecnología } & \multicolumn{2}{c}{ Perú } \\
\hline Hidráulica & 4942 & 4449 \\
Gas & 4361 & 1050 \\
Diesel & 2439 & 688 \\
Carbón & 140 & - \\
Solar & 285 & 24 \\
Eólica & 375 & 17 \\
Biomasa & 38 & 138 \\
Máxima Demanda & 6885 & 3924 \\
Interconexión & 200 & 200 \\
Margen de reserva (\%) & 0.45 & 0.37 \\
\hline
\end{tabular}

\section{METODOLOGÍA}

Para analizar el efecto de la interconexión de dos sistemas eléctricos se desarrolló un modelo que consideró los beneficios y riesgos de las diferentes 
políticas a implementar, así como el desarrollo dinámico del parque de generación y transmisión, Ver Fig. 1.

El modelo propuesto consta de dos submodelos: un modelo para el despacho de corto plazo y evaluación de inversiones y un modelo de expansión del sistema de largo plazo, los cuales interactúan y se retroalimentan de manera permanente.

La simulación comienza con el modelo de despacho, que proporciona la cantidad y costos marginales de la energía suministrada por cada planta de generación, para cubrir la demanda en cada uno de los países; así como, si los países están interconectados, la energía importada/exportada y las rentas de congestión. A continuación, dicha información es ingresada al modelo de expansión de capacidad y es el punto de partida para la simulación en dicho modelo, el cual sobre la base de indicadores de rentabilidad calcula la incorporación de nuevas plantas de generación por tipo de tecnología; así como, la expansión o no de la línea de interconexión. Finalmente, dicha información es retroalimentada al modelo de despacho para que inicialice nuevamente los cálculos para el siguiente periodo evaluado.

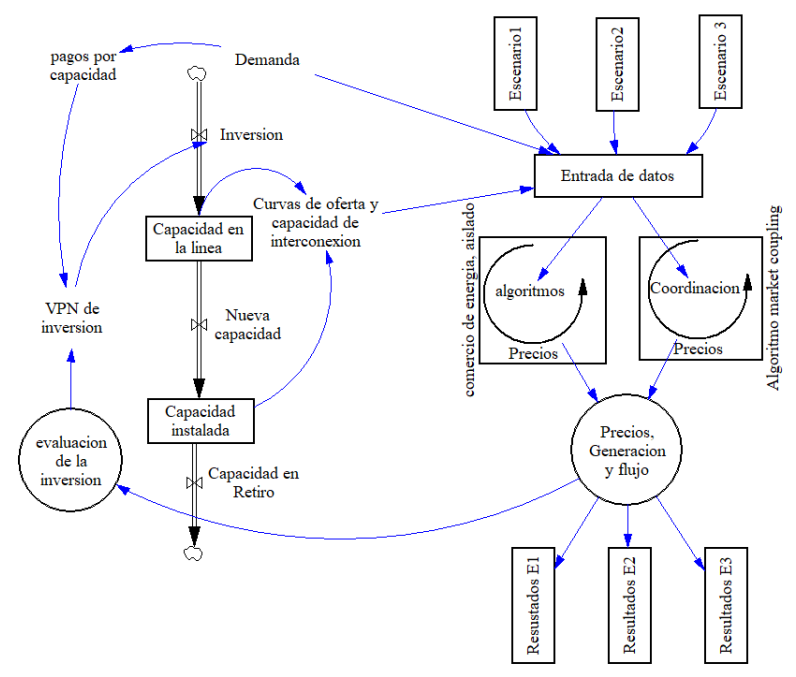

Fig. 1. Metodología Propuesta.

\subsection{MODELO DE DESPACHO Y CÁLCULO DEL VPN}

El modelo se basa en un algoritmo de despacho por orden de mérito de corto plazo que simula el despacho de energía por hora, tanto para su operación en forma aislada, con interconexión y con interconexión ilimitada, para ello toma en cuenta los principios de acoplamiento de mercado y desarrolla los siguientes procesos:

- Calcula la generación por fuente de cada país, en términos de energía eléctrica generada y costos marginales, flujos por las líneas de interconexión y rentas de congestión.

- Evalúa las inversiones, y calcula el valor presente neto (VPN) de las inversiones por cada tecnología.
El modelo de programación del despacho económico se formula de acuerdo a la ecuación (1).

$\min \sum_{i=1}^{N G} b_{i}\left(\sum_{j=1}^{N H} G_{i j}\right)$

Sujeto a:

$\begin{array}{ll}\sum_{i=1}^{N G} G_{i j}=D_{j} & ; \mathrm{j}=1 \ldots ., \mathrm{NH} \\ G_{i j} \leq \operatorname{Pax}_{i} & ; \mathrm{i}=1 \ldots ., \mathrm{NG} \quad \mathrm{j}=1 \ldots, \mathrm{NH} \\ G_{i j}=0 & ; \mathrm{i}=1 \ldots ., \mathrm{NG} \quad \mathrm{j}=1 \ldots, \mathrm{NH}\end{array}$

Donde:

$b_{i} \quad$ : Costo variable de operación de la central i

$\operatorname{Pmax}_{i}$ : Capacidad de la central i

$G_{i j} \quad$ : Generación de la central i en el bloque j

$D_{j} \quad$ : Demanda en la hora j

NG : número de generadores

$\mathrm{NH}$ : número de horas

La solución óptima a este problema es equivalente a ordenar los generadores de menor a mayor costo variable en una lista de mérito, el operador del sistema despacha las unidades en ese orden hasta cubrir la demanda en cada período. Este algoritmo fue implementado en Matlab.

\subsection{MODELO DE EXPANSIÓN}

El modelo de expansión simula la evolución del parque de generación, así como la disponibilidad que tendrán durante su operación. Para ello se han estructurado 4 módulos los cuales se listan a continuación:

a) Módulo de evaluación de la inversión

La capacidad en proceso incluye la inversión decidida, la capacidad en el proceso de permisos y la capacidad en construcción, que se separan en el modelo para tener en cuenta las diferentes demoras.

b) Módulo del ciclo de vida de la capacidad instalada

Se evalúa el ciclo de vida del parque de generación y la línea de interconexión, desde el momento de la inversión hasta el momento del retiro de la unidad, La capacidad instalada determina las curvas de oferta y la capacidad de la línea de interconexión, que son los datos que se exportan al modelo de despacho para calcular las cantidades de energía generada y costos marginales. Este módulo también evalúa un análisis de esquemas de pagos por capacidad que podrían ser implementados en los escenarios evaluados.

c) Módulo de regulación de reservorio 
Este módulo simula la regulación de los reservorios disponibles en ambos países, y es un dato de entrada esencial para el algoritmo de despacho, pues provee la información de la capacidad de generación que se tienen en forma diaria. El detalle de las ecuaciones empleadas en los módulos descritos está basado en [14] al [16] se presenta en el apéndice B. Para la implementación del modelo se utilizó el software Vensim DSS, Versión 7.3.5.

\section{RESULTADOS}

Con la finalidad de analizar el impacto que tendrá la interconexión entre Perú y Ecuador en la evolución del parque de generación de electricidad en cada país, se han definido y simulado los escenarios presentados en la Tabla II.

TABLA II

Descripción de Escenarios

\begin{tabular}{l|l}
\hline \multicolumn{1}{c}{ Escenario } & \multicolumn{1}{c}{ Descripción } \\
\hline \multirow{2}{*}{ Aislado } & $\begin{array}{l}\text { No se prevé interconexión entre ambos } \\
\text { países. } \\
\text { Se prevé el ingreso de la interconexión de } \\
500 \mathrm{MW} \text { entre Colombia y Ecuador. Se } \\
\text { puede agregar más capacidad de } \\
\text { transmisión de acuerdo con la rentabilidad } \\
\text { esperada, } \\
\text { Interconexión } \\
\text { Interconexión se prevé que las importaciones y } \\
\text { límite }\end{array}$ \\
& $\begin{array}{l}\text { exportaciones no están limitadas por la } \\
\text { capacidad de la interconexión, lo que } \\
\text { implica un despacho conjunto. }\end{array}$ \\
\hline
\end{tabular}

El escenario de interconexión comienza con la capacidad de interconexión de $500 \mathrm{MW}$ que se prevé instalar en los próximos años (se asume el año 2022), y las decisiones de expansión se toman en función del VPN de la inversión, suponiendo que el propietario de la línea de interconexión pueda cobrar las rentas de congestión. Aunque, las rentas de congestión no siempre son recaudadas por el propietario de la red; sin embargo, es una buena aproximación ya que los comités operadores de los sistemas pueden considerar los ingresos que generan las rentas de congestión para la expansión de la interconexión cuando negocian acuerdos bilaterales.

El escenario de operación aislada es el que representa el estado actual de ambos sistemas, ya que, si bien es cierto existe una línea de interconexión de 220 $\mathrm{kV}$, esta no opera de forma continua y solo sirve para el intercambio puntual de excedentes de generación.

El escenario de interconexión sin límite representa un despacho conjunto, lo cual no es realista, sin embargo, se ha modelado a fin de que sirva de punto de comparación y que ayude a explicar las consecuencias de la interconexión y de luces de la coherencia de los resultados obtenidos.

Los resultados de la simulación presentan que los costos marginales promedios mensuales en Ecuador tiene una gran correlación con su hidrología ya que en los últimos años han ingresado a su sistema una importante cantidad de centrales hidroeléctricas de pasada las cuales influenciaran a la baja en los costos marginales de los próximos años. Ver Fig. 2. Asimismo, se observa que en los primeros cinco años de simulación no hay un efecto importante ya que la línea de interconexión recién se pone en funcionamiento el año 2022, lo cual tiene un efecto inmediato en la subida de costos marginales en Ecuador, en el escenario de interconexión sin límite este efecto es mayor en comparación con los escenarios de interconexión y el escenario aislado, esto debido a que en el escenario de interconexión sin límites los costos marginales de ambos sistemas eléctricos siempre van a converger como si se tratase de un sistema único. Por su parte, el sistema peruano una vez se realiza la interconexión en el año 2022, experimenta una reducción de costos marginales, como se presenta en la Fig. 3.



Fig. 2. Evolución de los costos marginales de generación en Ecuador. 


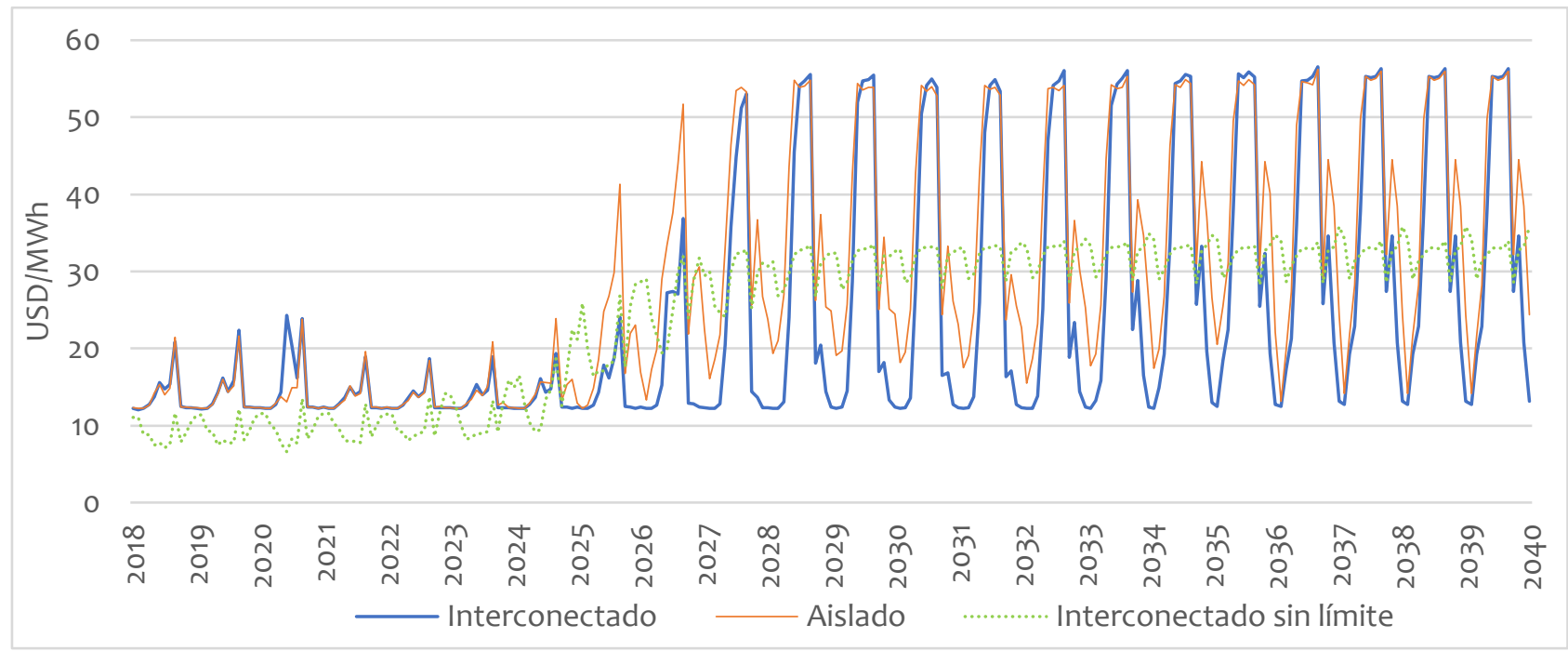

Fig. 3. Evolución en los costos marginales de generación en Perú.

En el escenario de interconexión Ecuador experimenta un ligero aumento en sus costos marginales como resultado de las exportaciones a Perú, que resultan en una disminución significativa en los costos marginales de Perú, especialmente en los meses de alta disponibilidad de agua en Ecuador, como se observa en la Fig. 3.

La razón es que Ecuador no cuenta con la capacidad de regular sus reservorios pues la mayor parte de las grandes centrales hidroeléctricas son de pasada, esto origina que solo pueda exportar en época de lluvias. Este efecto se verá amplificado cuanto mayor sea la capacidad de la línea de interconexión, ya que la demanda de exportación representaría una parte importante de su demanda total.

Además, se debe tener en cuenta que, en el escenario de interconexión, la red se expande significativamente hasta llegar a tener una capacidad de 2200 MW. Ver Fig. 4. Asimismo, La Fig. 5, presenta la dirección de los flujos de electricidad, la cual se alterna según la hidrología estacional, es decir el flujo va de Ecuador a Perú en la época de avenida en Ecuador y va de Perú a Ecuador en la época de avenida en Perú. Por lo tanto, en este escenario, los países se complementan entre sí en términos de disponibilidad de recursos hídricos.

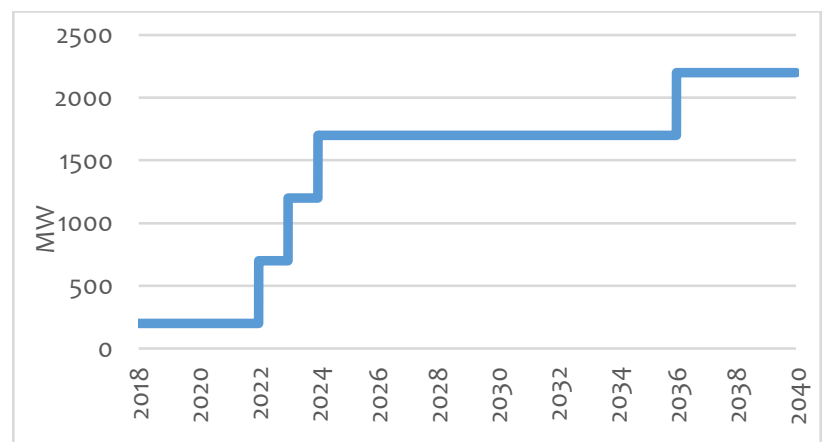

Fig. 4. Ampliación de la Capacidad de la Línea de Interconexión Perú - Ecuador.



Fig. 5. Flujo de energía en el escenario de interconexión 


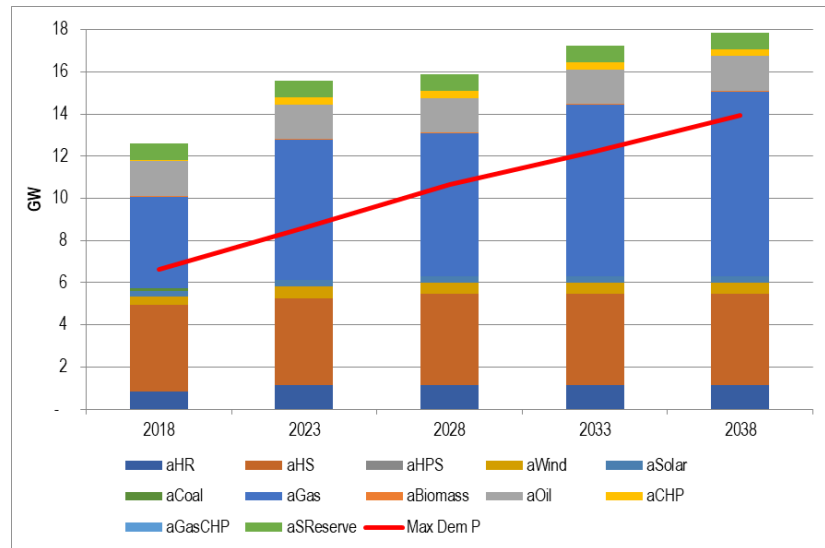

a)

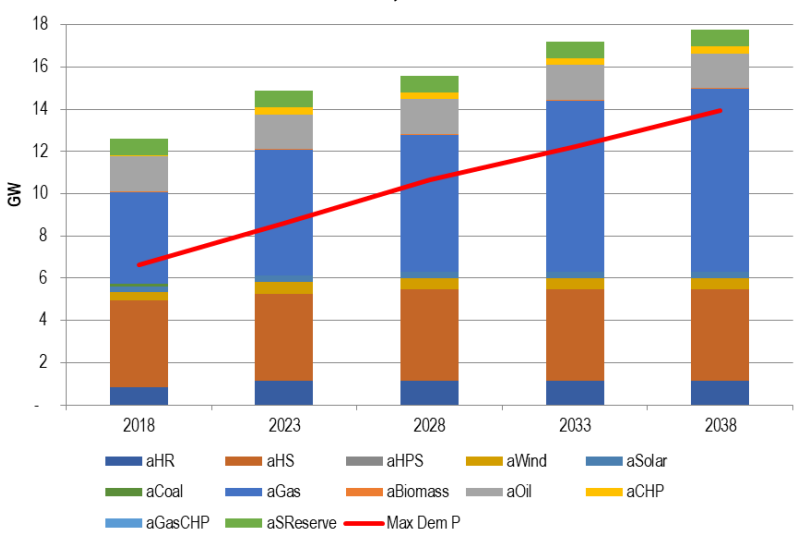

c)

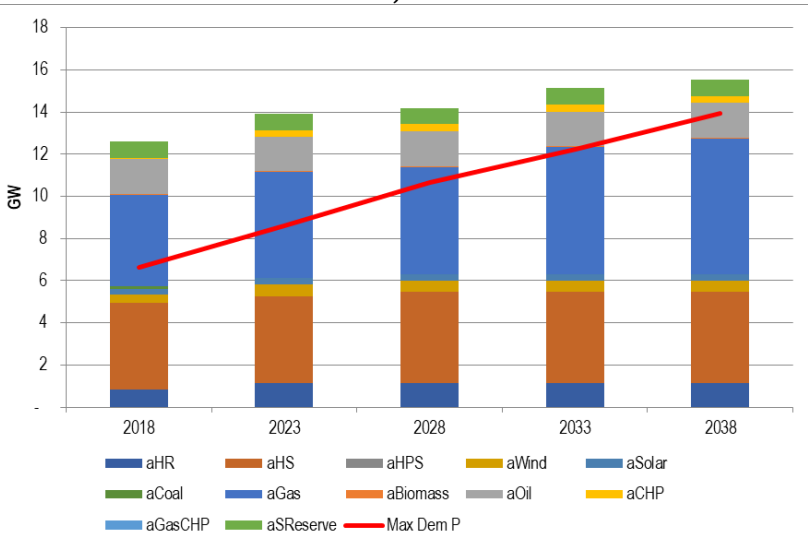

e)

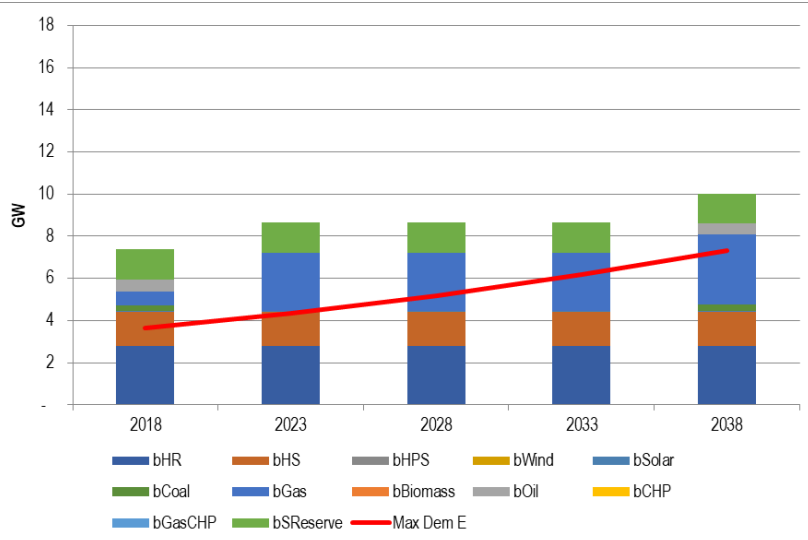

b)

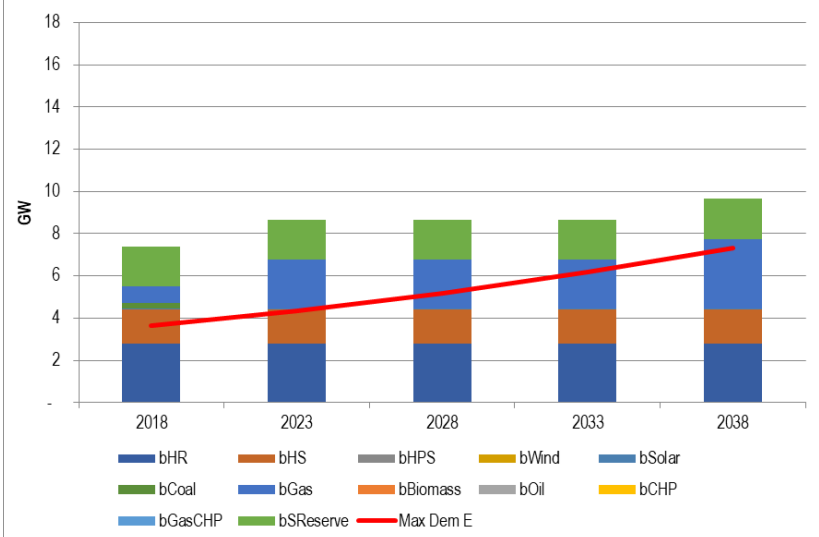

d)



f)

Fig. 6. Evolución de la Capacidad Instalada por Tipo de Tecnología. (a) Perú Aislado, (b) Ecuador Aislado, (c) Perú interconectado, (b) Ecuador Interconectado, (a) Perú Interconexión sin límite, (b) Ecuador Interconexión sin límite.

Sin embargo, los beneficios del escenario de interconexión para Ecuador son cuestionables, ya que mientras por un lado los generadores obtendrán rentas más altas como resultado del aumento de los precios y ventas de la exportación (ver la Fig. 2), por otro, costos marginales más altos se traducirán en tarifas más caras que los consumidores tendrán que pagar.

Desde el punto de vista de las decisiones de inversión en capacidad de generación, el estado actual del parque de generación tanto en Perú como en Ecuador, presenta altos márgenes de reserva, lo que responde a decisiones de inversión que no obedecieron a señales de mercado sino a políticas de estado en cada país. En el caso de Perú se llevaron a cabo licitaciones de energía provenientes de fuentes hidroeléctrica, la implementación del nodo energético del sur con una capacidad de $1000 \mathrm{MW}$ a base de gas natural; asimismo, sus leyes permiten la libre declaración del precio del gas natural para generación. En el caso de Ecuador se ha implementa una política de incorporación de centrales hidroeléctricas por $2746 \mathrm{MW}$. Estos hechos han originado que el costo marginal en ambos países sea relativamente bajo, y que no permitan que los nuevos proyectos de generación que podrían instalarse no logren recuperar su inversión, con lo cual la incorporación de nuevos proyectos de generación sea postergada durante varios años. $\mathrm{Y}$ aunque expandir la 
capacidad hidroeléctrica puede parecer una mejor solución dada la complementariedad hídrica entre ambos países, los bajos costos marginales que se presentan en ambos sistemas hacen que la las primeras plantes en entrar a dichos sistemas sean las centrales a gas natural. Como se observa en la Fig. 6.

\section{CONCLUSIONES}

El modelo presentado en este documento ayuda a comprender los nuevos desafíos para los reguladores del mercado eléctrico como consecuencia de la interconexión. Los resultados de la simulación muestran que la integración del mercado de electricidad puede traer beneficios en términos de menores costos de suministro y mejor uso de los recursos; sin embargo, estos beneficios dependen en gran medida del grado de interconexión y varían de un país a otro, dependiendo, entre otras cosas, del estado actual de sus parques de generación de electricidad y los márgenes de reserva con que cuenten.

- Si bien el impacto de la integración es más fuerte en el país importador, objetivos como la maximización del bienestar social total tienden a favorecer al país importador. Pero sacrificar el bienestar del país exportador en favor de un vecino, este hecho sería poco aceptable para el país exportador. Por lo tanto, se necesita un mecanismo de redistribución para aprovechar las oportunidades de integración y evitar que un país pierda. Sin embargo, diseñar un mecanismo para compensar al perdedor, sin afectar negativamente las señales del mercado, es una tarea muy compleja.

- En algunos casos, existe una compensación entre precios más bajos e independencia del país vecino, lo que plantea la cuestión de la confianza en las relaciones internacionales. Dado que la electricidad es un servicio estratégico con un impacto crucial en la economía nacional, la dependencia excesiva de fuentes externas puede no ser aceptable. Esto es particularmente importante en la región en estudio, debido a la falta de estabilidad política y los graves problemas de orden público cerca de las fronteras, que pueden afectar las transacciones internacionales de electricidad.

\section{AGRADECIMIENTOS}

Los autores brindan un agradecimiento especial al Fondo Nacional de Desarrollo Científico, Tecnológico y de Innovación Tecnológica (Convenio $n^{\circ}$ 207-2015FONDECYT) por la beca de doctorado en ciencias con mención en energética.
APENDICE A: TABLA DE ACRONIMOS

\begin{tabular}{|c|c|}
\hline \multicolumn{2}{|r|}{$\begin{array}{l}\text { TABLA III } \\
\text { Acrónimos }\end{array}$} \\
\hline Acrónimo & Descripción \\
\hline $\mathrm{ARCH}$ & $\begin{array}{l}\text { Agencia de Regulación y Control } \\
\text { Hidrocarburífero }\end{array}$ \\
\hline COES & $\begin{array}{l}\text { Comité de Operación Económica del } \\
\text { Sistema }\end{array}$ \\
\hline $\mathrm{kV}$ & Kilo Voltios \\
\hline ME & Mercado de solo energía \\
\hline MINEM & Ministerio de Energía y Minas \\
\hline MRC & $\begin{array}{l}\text { Mecanismos de remuneración de } \\
\text { capacidad }\end{array}$ \\
\hline MW & Mega Watts \\
\hline OSINERGMIN & $\begin{array}{l}\text { Organismo Supervisor de la Inversión en } \\
\text { Energía y Minería }\end{array}$ \\
\hline SEIN & Sistema Eléctrico Interconectado Nacional \\
\hline SUNAT & $\begin{array}{l}\text { Superintendencia Nacional de Aduanas } \\
\text { y de Administración Tributaria }\end{array}$ \\
\hline USD/MWh & $\begin{array}{l}\text { Dólares norteamericanos por Megawhatt- } \\
\text { hora }\end{array}$ \\
\hline VPN & Valor presente neto \\
\hline
\end{tabular}

\section{APENDICE B: ECUACIONES EMPLEADAS EN LOS MÓDULOS DEL MODELO DE EXPANSIÓN}

Cuando el VPN es positivo la inversión será realizada. La tarifa de inversión (I) es definida como el mínimo entre la magnitud de inversión (Mdl) y el potencial de expansión (PE) según los recursos disponibles de cada país

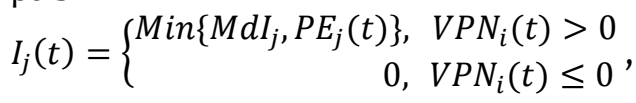

DI: decidir invertir

$D I_{j}(t)=\int_{t 0}^{t}\left[T I_{j}(s)-I P_{j}(s)\right] d s$,

IP: inicio del permiso

$I P_{j}(t)=$

$\left\{\begin{array}{c}\left.D I_{j}(t), \text { si }\left[\left(D M(t)=2 \text { y } I_{j}(t)=0\right\} o D I_{j}(t) \geq M S o I_{j}\right)\right] \\ 0, \text { otrocaso }\end{array}\right.$

CPP: Capacidad en proceso de permiso

$C P P_{j}(t)=\int_{t 0}^{t}\left[I P_{j}(s)-O P_{j}(s)\right] d s$,

$O P_{j}(t)=I P_{j}\left(t-p d_{j}\right)$,

$C U C_{j}(t)=\int_{t 0}^{t}\left[O P_{j}(s)-C_{j}(s)\right] d s+C U C_{j}(t),(\mathrm{B} 6)$

$C_{j}(t)=O P_{j}\left(t-c d_{j}\right)+P C_{j}(t)$,

$I C A_{1, j}(t)=\int_{t 0}^{t}\left[C_{j}(s)-I C A R_{1, j}(s)-R C_{1, j}(s)\right] d s+$

IC $A_{1, j}\left(t_{0}\right)$,

$I P_{j}(t)=\left\{\begin{array}{c}\frac{I C A_{1, j}\left(t_{0}\right)-\sum_{t} P R_{j}(t)}{l t_{j}}+P R_{j}(t), t \leq l t_{j}, \\ 0, t>l t_{j}\end{array}\right.$ 


\section{REFERENCIAS}

[1] T. Jamasb y M. Pollitt, "Security of supply and regulation of energy networks", Energy Policy, vol. 36, no. 12, pp. 4584-4589, dec. 2008. [2] S. Stoft, "Problems of transmission investment in a deregulated power market" in Competitive electricity markets and sustainability, 2006, pp. 87-130.

[3] P. Cramton, A. Ockenfels y S. Stoft, "Capacity market fundamentals", Economics of Energy \& Environmental Policy, vol. 2, no. 2, pp. 27-46, sep 2013.

[4] P. Mastropietro, P. Rodilla y C. Batlle, "National capacity mechanisms in the european internal energy market: Opening the doors to neighbours", Energy policy, vol. 28, pp. 38-47, jul 2015.

[5] D. Finon, "Capacity Mechanisms and Cross-border Participation: the EU Wide Approach in Question. 2018", HAL, hal-01687454, jun. 2018.

[6] F. Jara et al., "Interconexión de Mercados Transfronterizos de Electricidad y la Necesidad de Políticas de Coordinación", Revista Tecnia, vol. 28, n 1, pp. 1-4, dic. 2018.

[7] R. Meyer y O. Gore, "Cross-border effects of capacity mechanisms: Do uncoordinated market design changes contradict the goals of the European market integration?", Energy Economics, vol. 51, pp. 9-20. Jun. 2015.

[8] S. Lorenczik, "Interaction effects of market failure and CRMs in interconnected electricity markets”, Energy Policy, vol. 135, 110961. Dec. 2019.

[9] J. M. Glachant, N. Rossetto y J. Vasconcelos, “Moving the electricity transmission system towards a decarbonised and integrated Europe: Missing pillars and roadblocks". Florence School of Regulation Research Report, 2017.

[10] F. Boffa, V. Pingali y D. Vannoni, "Increasing market interconnection: An analysis of the Italian electricity spot market", International Journal of Industrial Organization, vol. 28, pp. 311-322. Nov. 2010.
[11] P. C. Bhagwat, K. lychettira y L. J. de Vries, “Cross-border effects of capacity mechanisms", in: 2014 11th International Conference on the European Energy Market (EEM), pp. 1-5.

[12] M. Cepeda y D. Finon, "Generation capacity adequacy in interdependent electricity markets", Energy Policy, vol. 51, pp. 31283143. Feb. 2011.

[13] C. Elberg, "Cross-border effects of capacity mechanisms in electricity markets", Energiewirtschaftliches Institut an der Universitaet zu Koeln , EWI Working Papers 2014-11. 2014.

[14] C. Ochoa y O. Gore, "The Finnish power market: Are imports from Russia low-cost?”, Energy Policy, vol. 80, pp. 122-132, jun. 2015.

[15] C. Ochoa y A. Van Ackere, "Does size matter? Simulating electricity market coupling between Colombia and Ecuador", Renewable and Sustainable Energy Reviews, vol. 50, pp. 1108-1124, jun. 2015.

[16] C. Ochoa y O. Gore, "Winners and losers of market coupling", Energy, vol. 80, pp. 522-534, jun. 2016.

[17] O. Gore, E. Vanadzina y S. Viljainen, "Linking the energy-only market and the energy-plus-capacity market", Utilities Policy, vol. 38, pp. 52-61, 2016.

[18] G. Hawker y K. Bell, S. Gill, "Electricity security in the European Union - The conflict between national capacity mechanisms and the single market", Energy Research \& Social Science, vol. 24, pp. 51-58, 2017.

[19] S. Osorio y A. van Ackere, "From nuclear phase-out to renewable energies in the Swiss electricity market", Energy Policy, vol.93, pp. 822, 2016.

[20] K. Neuhoff, J. Diekmann, F. Kunz, S. Ruster, W. P. Schill y S. Schwenen, "A coordinated strategic reserve to safeguard the European energy transition”, Utilities Policy, vol. 41, pp. 252-263, 2016. 